\title{
The potential of salivary point of care diagnostics
}

\author{
Christina M. BONEBREAK ${ }^{1}$, Utkan DEMIRCI ${ }^{2}$, Winston Patrick KUO ${ }^{3}$
}

\begin{abstract}
1- Dental student, Harvard School of Dental Medicine, Boston, Massachusetts, 02115, USA. Harvard Catalyst, Laboratory for Innovative Translational Technologies, Harvard Medical School, Boston. Christina_Bonebreak@hsdm.harvard.edu

2- PhD, Director, Bio-Acoustic-MEM in Medicine Laboratory, Center for Biomedical Engineering, Assistant Professor, Department of Medicine, Brigham and Women's Hospital, Harvard Medical School, Boston, Massachusetts, 02115, USA. udemirci@rics.bwh.harvard.edu

3- DDS, SM, DMSc, Director, Harvard Catalyst, Laboratory for Innovative Translational Technologies, Harvard Medical School, Boston, 02115, USA; Assistant Professor, Department of Developmental Biology, Harvard School of Dental Medicine, Boston, Massachusetts, 02115, USA. wkuo@catalyst.harvard.edu
\end{abstract}

Point of care (POC) diagnostic technologies has gained much attention in the biomedical community as a methodology for early screening to diagnose and monitor the effectiveness of therapeutics (companion diagnostics) that is cost-effective and easy to use. The ability to utilize POC diagnostics to screen and quantify biomarkers associated with disease prognosis and predictive therapeutic response would assist dentists/ physicians in their treatment selection ${ }^{1}$.

The evolution of POC diagnostic technologies demonstrates that technology development is at a point in which it can address clinical needs. Simple chemical/ colorimetric methods were quickly developed into dipstick type formats that have been used by physicians at the first stage of patient assessment; an example of this is the urine analysis in a clinical setting. Urine samples are easily obtained non-invasively without requiring technical or a highly-trained person ${ }^{4}$. There also have been recent attempts to use finger-prick (minimally invasive) volumes of blood samples to diagnose and monitor infectious disease using on-chip microdevices ${ }^{2}$. While many variations of these devices have been utilized for blood-borne biomarkers of disease, the use of saliva as a diagnostic fluid has not gained much traction due to methodological and technological obstacles related to the fluid itself as the viscosity and presence of high abundant proteins (mucins) and microbial contaminations (if one is not targeting this population). Though, given its availability (and the fact most patients visit their dentist just as often as their primary care physician), the noninvasive nature of collection, relative low cost of testing, and proximity to oral tissues, saliva would be an ideal fluid for oral diagnostics. Advantages to this approach is that many diagnostic analytes in serum have been found in saliva and these observations give saliva the potential for use in therapeutic drug monitoring, as is being developed for epilepsy, and in the diagnosis of systemic disorders such as impaired cardiovascular function, renal disease, cancer, and viral and bacterial infections.

The benefits of early detection are critical to improving the outcome of treatment, survival rate, quality of life, and cost of care of many diseases. Limited screening tests exist for common oral diseases such as caries, periodontitis and oral cancer, resulting in many late- and end-stage diagnoses and poor prognoses.

The combination of using the novel use of microparticles isolated from saliva with microfluidic technologies hold promise for diagnosing disease, monitoring disease progression and evaluating therapeutic responses. Traditional diagnostic methods test for the presence of specific DNA or RNA molecules associated with a variety of disease states that require labor-intensive sample preparation that typically involves cell lysis, nucleic acid/ protein isolation, enrichment, purification and detection.
Advancements in POC diagnostic technology development permits micro- and nano-scale reaction volumes where all the steps can be incorporated on a "lab-on-a-chip", which is a miniaturized device, such as a cassette, that can then be inserted into a portable detection analyzer. Such devices also allow for the simultaneous evaluation of multiple analytes (multi-plexing) from a single fluid sample, which may be required for the detection and analysis of complex diseases such as periodontitis and oral cancer. For example, in monitoring the progression of periodontitis, the analysis of a panel of biomarkers associated with the inflammatory process (IL-1B, MMP-8, and ICTP) is shown to have significantly higher diagnostic value than the measurement of a single biomarker.

The use of saliva as a diagnostic fluid presents several challenges related to low abundant analytes that can be addressed by a two or three component microfluidic device. The amount of analytes tend to be much lower in concentration in saliva when compared to that of serum that can be mitigated by employing analyte enrichment techniques or utilizing a more sensitive detection method such as using quantum dot technology (nanoparticles display unique optical and electrical properties).

In summary, there are numerous advantages to using microfluidic salivary diagnostics from the non-invasive method of saliva collection that causes minimal discomfort and anxiety, which can improve patient compliance for testing to the benefits of lab-on-a-chip device for clinical diagnostics to include scalability, low-cost, disposable devices, the use of small sample volumes, limited exposure to and use of chemical reagents, automated process and rapid processing times. Additionally, on the global health related issues, portable, fully-automated analyzing systems can provide considerable benefit to the healthcare of persons in rural or remote regions which may lack sophisticated laboratory facilities or highly-trained personnel. The parallel progress in salivary biomarker discovery and microfluidic processing methodologies has made it possible for the development of lab-on-a-chip devices for potential diagnosis and monitoring of many oral and systemic diseases ${ }^{3}$. Continued research in these fields will lead to the widespread adoption of salivary diagnostics in the near future.

\section{REFERENCES}

1- Giljohann DA, Mirkin CA. Drivers of biodiagnostic development. Nature. 2009;462(7272):461-4.

2- Kim YG, Moon S, Kuritzkes DR, Demirci U. Quantum dotbased HIV capture and imaging in a microfluidic channel. Biosens Bioelectron. 2009;25(1):253.

3- Spielmann N, Wong DT. Saliva: diagnostics and therapeutic perspectives. Oral Dis. 2011;17(4):345-54.

4- Wallace JF, Pugia MJ, Lott JA, Luke KE, Shihabi ZK, Sheehan M, et al. Multisite evaluation of a new dipstick for albumin, protein, and creatinine. J Clin Lab Anal. 2001;15(5):231-5. 